\title{
QUANTUM LENS SPACES AND GRAPH ALGEBRAS
}

\author{
Jeong Hee Hong And Wojciech Szymański
}

\begin{abstract}
We construct the $C^{*}$-algebra $C\left(L_{q}\left(p ; m_{1}, \ldots, m_{n}\right)\right)$ of continuous functions on the quantum lens space as the fixed point algebra for a suitable action of $\mathbb{Z}_{p}$ on the algebra $C\left(S_{q}^{2 n-1}\right)$, corresponding to the quantum odd dimensional sphere. We show that $C\left(L_{q}\left(p ; m_{1}, \ldots, m_{n}\right)\right)$ is isomorphic to the graph algebra $C^{*}\left(L_{2 n-1}^{\left(p ; m_{1}, \ldots, m_{n}\right)}\right)$. This allows us to determine the ideal structure and, at least in principle, calculate the $K$ groups of $C\left(L_{q}\left(p ; m_{1}, \ldots, m_{n}\right)\right)$. Passing to the limit with natural imbeddings of the quantum lens spaces we construct the quantum infinite lens space, or the quantum EilenbergMacLane space of type $\left(\mathbb{Z}_{p}, 1\right)$.
\end{abstract}

\section{Introduction.}

Classical lens spaces $L\left(p ; m_{1}, \ldots, m_{n}\right)$ are defined as the orbit spaces of suitable free actions of finite cyclic groups on odd dimensional spheres (e.g., see $[\mathbf{1 3}]$ ). In the present article, we define and investigate their quantum analogues. The $C^{*}$-algebras of continuous functions on the quantum lens spaces were introduced earlier by Matsumoto and Tomiyama in [18], but our construction leads to different (in general) algebras. (The very special case of the quantum 3-dimensional real projective space was investigated by Podleś $[\mathbf{2 0}]$ and Lance [17], in the context of the quantum $S O(3)$ group.) The starting point for us is the $C^{*}$-algebra $C\left(S_{q}^{2 n-1}\right), q \in(0,1)$, of continuous functions on the quantum odd dimensional sphere. If $n=2$ then $C\left(S_{q}^{3}\right)$ is nothing but $C\left(S U_{q}(2)\right)$ of Woronowicz [27]. The construction in higher dimensions is due to Vaksman and Soibelman [26], and from a somewhat different perspective to Nagy [19]. (See also the closely related construction of representations of the twisted canonical commutation relations due to Pusz and Woronowicz $[\mathbf{2 1}]$.) We define the $C^{*}$-algebra $C\left(L_{q}\left(p ; m_{1}, \ldots, m_{n}\right)\right)$ of continuous functions on the quantum lens space as the fixed point algebra for a suitable action of the finite cyclic group $\mathbb{Z}_{p}$ on $C\left(S_{q}^{2 n-1}\right)$. This definition depends on the deformation parameter $q \in(0,1)$, as well as on positive integers $p \geq 2$ and $m_{1}, \ldots, m_{n}$. We normally assume that each of $m_{1}, \ldots, m_{n}$ is relatively prime to $p$. On the classical level, this guarantees freeness of the action. In the special case $p=2, m_{1}=\cdots=m_{n}=1$ we recover 
quantum odd dimensional real projective spaces, defined and investigated in our earlier article [11].

The key technical result on which this article depends is Theorem 4.4 of [11], which gives an explicit isomorphism between $C\left(S_{q}^{2 n-1}\right)$ and the $C^{*}$-algebra $C^{*}\left(L_{2 n-1}\right)$ corresponding to the directed graph $L_{2 n-1}$. Thus, $C\left(L_{q}\left(p ; m_{1}, \ldots, m_{n}\right)\right)$ is isomorphic to the fixed point algebra $C^{*}\left(L_{2 n-1}\right)^{\Lambda}$, corresponding to a suitable action $\Lambda: \mathbb{Z}_{p} \rightarrow \operatorname{Aut}\left(C^{*}\left(L_{2 n-1}\right)\right)$. This allows us to employ in our investigations of the quantum lens spaces the huge and comprehensive machinery developed for dealing with Cuntz-Krieger algebras of directed graphs (cf. $[6,5,16,12,15,14,2,9,24,10,22,25,1]$ and references there).

In order to understand the fixed point algebra $C^{*}\left(L_{2 n-1}\right)^{\Lambda}$ we first look at the crossed product $C^{*}\left(L_{2 n-1}\right) \rtimes_{\Lambda} \mathbb{Z}_{p}$. By virtue of the results of [14] this crossed product itself is isomorphic to the $C^{*}$-algebra of the skew product graph $L_{2 n-1} \times_{c} \mathbb{Z}_{p}$, corresponding to a suitable labelling $c$ of the edges of $L_{2 n-1}$ by elements of $\mathbb{Z}_{p}$. The action $\Lambda$ is saturated and, hence, $C^{*}\left(L_{2 n-1}\right)^{\Lambda}$ is isomorphic to a full corner of $C^{*}\left(L_{2 n-1} \times_{c} \mathbb{Z}_{p}\right)$. This allows us, at least in principle, to calculate the $K$-groups of $C\left(L_{q}\left(p ; m_{1}, \ldots, m_{n}\right)\right)$.

Our main result, Theorem 2.5, shows that $C\left(L_{q}\left(p ; m_{1}, \ldots, m_{n}\right)\right)$ itself is isomorphic to the graph algebra $C^{*}\left(L_{2 n-1}^{\left(p ; m_{1}, \ldots, m_{n}\right)}\right)$, corresponding to a finite graph $L_{2 n-1}^{\left(p ; m_{1}, \ldots, m_{n}\right)}$. As a corollary, we easily deduce the ideal structure of $C\left(L_{q}\left(p ; m_{1}, \ldots, m_{n}\right)\right)$. We believe that on the basis of Theorem 2.5 one should be able to determine isomorphisms between the $C^{*}$-algebras of continuous functions on the quantum lens spaces, but this work is not carried in the present article.

\section{Preliminaries.}

1.1. Definition. We recall the definition of the $C^{*}$-algebra corresponding to a directed graph $[\mathbf{9}]$. Let $E=\left(E^{0}, E^{1}, r, s\right)$ be a directed graph with (at most) countably many vertices $E^{0}$ and edges $E^{1}$, and range and source functions $r, s: E^{1} \rightarrow E^{0}$, respectively. $C^{*}(E)$ is, by definition, the universal $C^{*}$-algebra generated by families of projections $\left\{P_{v} \mid v \in E^{0}\right\}$ and partial isometries $\left\{S_{e} \mid e \in E^{1}\right\}$, subject to the following relations:

(G1) $P_{v} P_{w}=0$ for $v, w \in E^{0}, v \neq w$.

(G2) $S_{e}^{*} S_{f}=0$ for $e, f \in E^{1}, e \neq f$.

(G3) $S_{e}^{*} S_{e}=P_{r(e)}$ for $e \in E^{1}$.

(G4) $S_{e} S_{e}^{*} \leq P_{s(e)}$ for $e \in E^{1}$.

(G5) $P_{v}=\sum_{e \in E^{1}: s(e)=v} S_{e} S_{e}^{*}$ for $v \in E^{0}$, provided $\left\{e \in E^{1} \mid s(e)=v\right\}$ is finite and nonempty. 
Universality in this definition means that if $\left\{Q_{v} \mid v \in E^{0}\right\}$ and $\left\{T_{e} \mid e \in E^{1}\right\}$ are families of projections and partial isometries, respectively, satisfying Conditions (G1)-(G5), then there exists a $C^{*}$-algebra homomorphism from $C^{*}(E)$ to the $C^{*}$-algebra generated by $\left\{Q_{v} \mid v \in E^{0}\right\}$ and $\left\{T_{e} \mid e \in E^{1}\right\}$ such that $P_{v} \mapsto Q_{v}$ and $S_{e} \mapsto T_{e}$ for $v \in E^{0}, e \in E^{1}$.

It follows from the universal property that there exists the gauge action $\gamma: \mathbb{T} \rightarrow \operatorname{Aut}\left(C^{*}(E)\right)$ such that $\gamma_{t}\left(P_{v}\right)=P_{v}$ and $\gamma_{t}\left(S_{e}\right)=t S_{e}$, for all $v \in E^{0}$, $e \in E^{1}, t \in \mathbb{T}$.

1.2. $K$-theory. The $K$-theory of a graph algebra $C^{*}(E)$ can be calculated as follows: Let $V_{E}$ be the collection of all those vertices which are not sinks and emit finitely many edges, and let $\mathbb{Z} V_{E}$ and $\mathbb{Z} E^{0}$ be free abelian groups on free generators $V_{E}$ and $E^{0}$, respectively. We have

$$
\begin{aligned}
& K_{0}\left(C^{*}(E)\right) \cong \operatorname{coker}\left(K_{E}\right), \\
& K_{1}\left(C^{*}(E)\right) \cong \operatorname{ker}\left(K_{E}\right),
\end{aligned}
$$

where $K_{E}: \mathbb{Z} V_{E} \rightarrow \mathbb{Z} E^{0}$ is the map defined on generators as

$$
K_{E}(v)=\left(\sum_{e \in E^{1}: s(e)=v} r(e)\right)-v .
$$

(See [5, Proposition 3.1], [16, Corollary 6.12], [22, Theorem 3.2], [24, Proposition 2] and [7, Theorem 3.1].)

1.3. Ideals. We assume that $E$ is a row-finite graph (i.e., each vertex of $E$ emits only finitely many edges) without sinks, since this is all we need in the present article. At first we describe closed 2-sided ideals of $C^{*}(E)$ invariant under the gauge action, as well as the corresponding quotients $[\mathbf{5}, \mathbf{1 2}, \mathbf{1 6}, \mathbf{2}, \mathbf{1}, \mathbf{1 0}]$. To this end we consider hereditary and saturated subsets of $E^{0}$. A subset $X \subseteq E^{0}$ is hereditary and saturated if the following two conditions are satisfied:

(HS1) If $v \in X, w \in E^{0}$ and there exists a path from $v$ to $w$ then $w \in X$.

(HS2) If $v \in E^{0}$ and for each $e \in E^{1}$ with $s(e)=v$ we have $r(e) \in X$, then $v \in X$.

We denote by $\Sigma_{E}$ the collection of all hereditary and saturated subsets of $E^{0}$. Any hereditary and saturated set $X$ gives rise to a gauge invariant ideal generated by $\left\{P_{v} \mid v \in X\right\}$ and denoted $J_{X}$. The quotient $C^{*}(E) / J_{X}$ is naturally isomorphic to $C^{*}(E / X)$, where $E / X$ denotes the restriction of the graph $E$ to $E^{0} \backslash X$. There exists a bijection between $\Sigma_{E}$ and the collection of all gauge invariant ideals of $C^{*}(E)$, given by the following two maps:

$$
X \mapsto J_{X}, \quad J \mapsto\left\{v \in E^{0} \mid P_{v} \in J\right\} .
$$


We now turn to the description of primitive ideals of the graph algebra $C^{*}(E)$ corresponding to a row-finite graph $E$ with no sinks $[\mathbf{1 2}, \mathbf{1 6}, \mathbf{2}, \mathbf{1}, \mathbf{1 0}]$. Key objects used in the classification of primitive ideals of graph algebras are maximal tails, defined as follows: A nonempty subset $M \subseteq E^{0}$ is called a maximal tail if the following three conditions are satisfied:

(MT1) If $v \in E^{0}, w \in M$ and there is a path in $E$ from $v$ to $w$ then $v \in M$.

(MT2) If $v \in M$ then there exists an edge $e \in E^{1}$ such that $s(e)=v$ and $r(e) \in M$.

(MT3) For any $v, w \in M$ there is a $y \in M$ such that there exist paths in $E$ from $v$ to $y$ and from $w$ to $y$.

The collection $\mathcal{M}(E)$ of all maximal tails is a disjoint union of its two subcollections $\mathcal{M}_{\gamma}(E)$ and $\mathcal{M}_{\tau}(E)$, defined as follows: A maximal tail $M$ belongs to $\mathcal{M}_{\gamma}(E)$ if and only if every vertex simple loop $\left(e_{1}, e_{2}, \ldots, e_{k}\right)$ (where $e_{i} \in E^{1}, r\left(e_{i}\right)=s\left(e_{i+1}\right), r\left(e_{k}\right)=s\left(e_{1}\right)$ and $r\left(e_{i}\right) \neq r\left(e_{j}\right)$ for $i \neq j$ ) whose all vertices $s\left(e_{i}\right)$ belong to $M$ has an exit $e \in E^{1}$ (that is, $s(e) \in\left\{s\left(e_{1}\right), \ldots s\left(e_{k}\right)\right\}$ but $\left.e \notin\left\{e_{1}, \ldots, e_{k}\right\}\right)$ with $r(e) \in M$. Otherwise $M$ belongs to $\mathcal{M}_{\tau}(E)$. It can be shown that each maximal tail from $\mathcal{M}_{\gamma}(E)$ gives rise to a primitive ideal of $C^{*}(E)$ invariant under the gauge action, and each maximal tail from $\mathcal{M}_{\tau}(E)$ gives rise to a circle of primitive ideals none of which is invariant under the gauge action. Let $\operatorname{Prim}\left(C^{*}(E)\right)$ denote the set of all primitive ideals of $C^{*}(E)$. If $E$ is a finite graph with no sinks then there exists a bijection

$$
\mathcal{M}_{\gamma}(E) \cup\left(\mathcal{M}_{\tau}(E) \times \mathbb{T}\right) \leftrightarrow \operatorname{Prim}\left(C^{*}(E)\right) .
$$

A complete description of the closure operation in the hull-kernel topology is also available. See $[\mathbf{1 2}, \mathbf{1 6}, \mathbf{2}, \mathbf{1}, \mathbf{1 0}]$ for the details.

We finish this section with the following lemma, which will be needed in the proof of Theorem 2.5. Recall that a closed 2-sided ideal $J$ of a $C^{*}$ algebra $A$ is essential if and only if for each nonzero element $a$ of $A$ we have $a J \neq\{0\}$.

Lemma 1.1. If $E$ is a row-finite graph and $X \neq \emptyset$ is a hereditary and saturated subset of $E^{0}$ then $J_{X}$ is an essential ideal of $C^{*}(E)$ if and only if for each vertex $v \in E^{0} \backslash X$ there exists a path in $E$ from $v$ to a vertex in $X$.

Proof. Suppose that for each vertex $v \in E^{0} \backslash X$ there exists a path in $E$ from $v$ to a vertex in $X$. With the gauge action $\gamma: \mathbb{T} \rightarrow \operatorname{Aut}\left(C^{*}(E)\right)$, the formula $\Gamma(b)=\int_{t \in \mathbb{T}} \gamma_{t}(b) d t$ (the integration with respect to the normalized Haar measure) defines a faithful conditional expectation from $C^{*}(E)$ onto the fixed point algebra $C^{*}(E)^{\gamma}$. Let $a \neq 0$ be an element of $C^{*}(E)$ and let $J^{\prime}$ be the closed 2-sided ideal of $C^{*}(E)$ generated by $\Gamma\left(a^{*} a\right)$. Since $J^{\prime}$ is a nonzero gauge invariant ideal there exists a vertex $v \in E^{0}$ such that $P_{v} \in J^{\prime}$ (cf. [2, Theorem 4.1]). If $\alpha$ is a path from $v$ to a vertex in $X$ then $S_{\alpha} \in J_{X}$ 
and $P_{v} S_{\alpha} \neq 0$. Consequently, $\{0\} \neq \Gamma\left(a^{*} a\right) J_{X}=\left(\int_{t \in \mathbb{T}} \gamma_{t}\left(a^{*} a\right) d t\right) J_{X}$. Thus, there exists a $t \in \mathbb{T}$ such that $\gamma_{t}\left(a^{*} a\right) J_{X} \neq\{0\}$. Since $\gamma_{t}\left(J_{X}\right)=J_{X}$ this implies $a J_{X} \neq\{0\}$. Therefore, the ideal $J_{X}$ is essential, as required. The converse implication is trivial.

1.4. Quantum odd dimensional spheres. For $n=1,2, \ldots$ and $q \in$ $(0,1)$ the $C^{*}$-algebra $C\left(S_{q}^{2 n-1}\right)$ of continuous functions on the quantum sphere $S^{2 n-1}$ is given in [26] as the universal $C^{*}$-algebra generated by elements $z_{1}, z_{2}, \ldots, z_{n}$, subject to the following relations:

$$
\begin{aligned}
z_{j} z_{i} & =q z_{i} z_{j} \quad \text { for } i<j, \\
z_{j}^{*} z_{i} & =q z_{i} z_{j}^{*} \quad \text { for } i \neq j, \\
z_{i}^{*} z_{i} & =z_{i} z_{i}^{*}+\left(1-q^{2}\right) \sum_{j>i} z_{j} z_{j}^{*} \quad \text { for } i=1, \ldots, n, \\
\sum_{i=1}^{n} z_{i} z_{i}^{*} & =I .
\end{aligned}
$$

It is shown in [11, Theorem 4.4] that the $C^{*}$-algebra $C\left(S_{q}^{2 n-1}\right)$ is isomorphic with a graph algebra $C^{*}\left(L_{2 n-1}\right)$. The graph $L_{2 n-1}$ has $n$ vertices $\left\{v_{1}, \ldots, v_{n}\right\}$ and $n(n+1) / 2$ edges $\bigcup_{i=1}^{n}\left\{e_{i, j} \mid j=i, \ldots, n\right\}$ with $s\left(e_{i, j}\right)=v_{i}$ and $r\left(e_{i, j}\right)=v_{j}$. It is a finite graph without sinks. For example, if $n=3$ then the corresponding graph $L_{5}$ looks as follows:

$L_{5}$

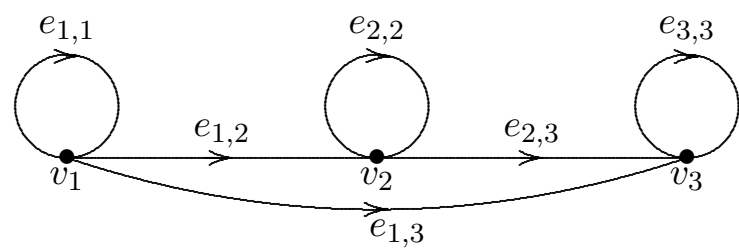

The isomorphism $\phi: C\left(S_{q}^{2 n-1}\right) \rightarrow C^{*}\left(L_{2 n-1}\right)$ is given explicitly on the generators as

$$
\begin{aligned}
\text { (5) } \phi: z_{n} \mapsto \sum_{k_{1}, \ldots, k_{n-1} \in \mathbb{N}} q^{k_{1}+\cdots+k_{n-1}} T\left(k_{1}, \ldots, k_{n-1}\right) S_{e_{n, n}} T\left(k_{1}, \ldots, k_{n-1}\right)^{*} \\
\text { (6) } \phi: z_{i} \mapsto \sum_{k_{1}, \ldots, k_{i} \in \mathbb{N}} q^{k_{1}+\cdots+k_{i-1}}\left(\sqrt{1-q^{2\left(k_{i}+1\right)}}-\sqrt{1-q^{2 k_{i}}}\right) \times \\
\quad \times T\left(k_{1}, \ldots, k_{i}\right)\left(\sum_{j=i}^{n} S_{e_{i, j}}\right) T\left(k_{1}, \ldots, k_{i}\right)^{*}
\end{aligned}
$$


for $i=1, \ldots, n-1$. Here for $k_{1}, \ldots, k_{i} \in \mathbb{N}$ we denoted

$$
T\left(k_{1}, \ldots, k_{i}\right)=\left(\sum_{j=1}^{n} S_{e_{1, j}}\right)^{k_{1}}\left(\sum_{j=2}^{n} S_{e_{2, j}}\right)^{k_{2}} \ldots\left(\sum_{j=i}^{n} S_{e_{i, j}}\right)^{k_{i}},
$$

an element of $C^{*}\left(L_{2 n-1}\right)$.

\section{Quantum lens spaces.}

We begin by recalling the definition of the classical lens spaces [13]. Namely, for $n=1,2, \ldots$ let $S^{2 n-1}=\left\{\left.\left(y_{1}, \ldots, y_{n}\right) \in \mathbb{C}^{n}\left|\sum_{i=1}^{n}\right| y_{i}\right|^{2}=1\right\}$ be the sphere of dimension $2 n-1$. We fix an integer $p \geq 2$ and $n$ integers $m_{1}, \ldots, m_{n}$. If $\theta=e^{2 \pi i / p}$ then

$$
\left(y_{1}, \ldots, y_{n}\right) \mapsto\left(\theta^{m_{1}} y_{1}, \ldots, \theta^{m_{n}} y_{n}\right)
$$

is a homeomorphism of $S^{2 n-1}$ which gives rise to an action of $\mathbb{Z}_{p}$, the cyclic group of order $p$, on $S^{2 n-1}$. The (generalized) lens space $L\left(p ; m_{1}, \ldots, m_{n}\right)$ of dimension $2 n-1$ is defined as the orbit space of this action. It is normally assumed that each of $m_{1}, m_{2}, \ldots, m_{n}$ is relatively prime to $p$. This assumption is equivalent to freeness of the action (8).

We now turn to the quantum case. With the sole exception of Lemma 2.1, we always assume that each of $m_{1}, m_{2}, \ldots, m_{n}$ is relatively prime to $p$. The universal property of $C\left(S_{q}^{2 n-1}\right)$ implies that the assignment

$$
\widetilde{\Lambda}: z_{i} \mapsto \theta^{m_{i}} z_{i},
$$

for $i=1, \ldots, n$, gives rise to an automorphism $\widetilde{\Lambda}$ of $C\left(S_{q}^{2 n-1}\right)$ of order $p$. For $q \in(0,1)$ we define the $C^{*}$-algebra $C\left(L_{q}\left(p ; m_{1}, \ldots, m_{n}\right)\right)$ of continuous functions on the quantum lens space as the fixed point algebra corresponding to this automorphism, i.e.,

$$
C\left(L_{q}\left(p ; m_{1}, \ldots, m_{n}\right)\right)=C\left(S_{q}^{2 n-1}\right)^{\widetilde{\Lambda}} .
$$

Let $\phi: C\left(S_{q}^{2 n-1}\right) \rightarrow C^{*}\left(L_{2 n-1}\right)$ be the isomorphism given by (5)-(6). Setting $\Lambda=\phi \widetilde{\Lambda} \phi^{-1}$ we get

$$
\begin{aligned}
\Lambda: P_{v_{i}} & \mapsto P_{v_{i}}, \\
\Lambda: S_{e_{i, j}} & \mapsto \theta^{m_{i}} S_{e_{i, j}},
\end{aligned}
$$

for $i=1, \ldots, n$ and $j=i, \ldots, n$. This gives

$$
C\left(L_{q}\left(p ; m_{1}, \ldots, m_{n}\right)\right)=C\left(S_{q}^{2 n-1}\right)^{\widetilde{\Lambda}} \cong C^{*}\left(L_{2 n-1}\right)^{\Lambda} .
$$

Actions of this type have been studied by Kumjian and Pask [14]. Let $c: L_{2 n-1}^{1} \rightarrow \mathbb{Z}_{p}$ be a labeling of the edges of $L_{2 n-1}$ such that $c\left(S_{e_{i, j}}\right)=m_{i}$. The skew product graph $L_{2 n-1} \times_{c} \mathbb{Z}_{p}$ is defined so that its vertices are $L_{2 n-1}^{0} \times \mathbb{Z}_{p}$ and its edges are $L_{2 n-1}^{1} \times \mathbb{Z}_{p}$ with $s\left(e_{i, j}, m\right)=\left(v_{i}, m-m_{i}\right)$ and 
$r\left(e_{i, j}, m\right)=\left(v_{j}, m\right)$, for $m \in \mathbb{Z}_{p}, i=1, \ldots, n$ and $j=i, \ldots, n$. We note that through each vertex of this graph passes precisely one vertex simple loop (composed of $p$ edges), and for any two vertices $\left(v_{i}, m\right),\left(v_{j}, k\right)$ there exists a path from $\left(v_{i}, m\right)$ to $\left(v_{j}, k\right)$ if and only if $i \leq j$. For example, if $n=2$, $p=3, m_{1}=1$ and $m_{2}=2$ then $L_{3} \times_{c} \mathbb{Z}_{3}$ looks as follows:

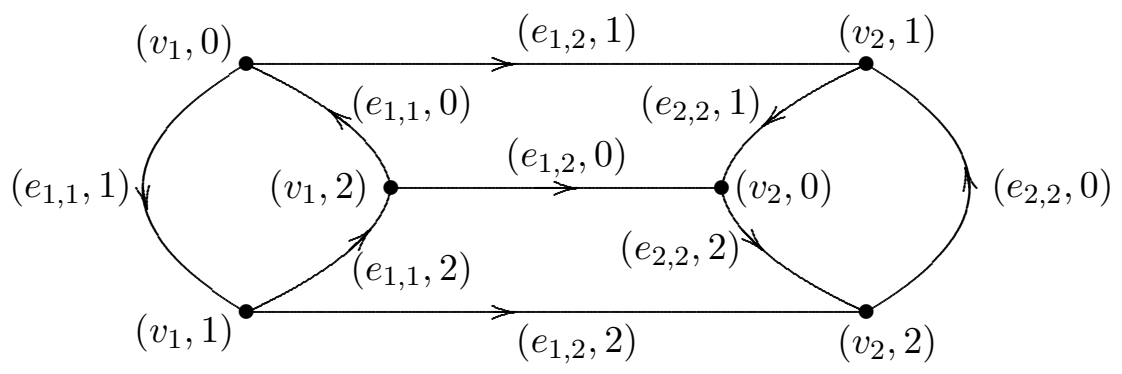

By virtue of $\left[\mathbf{1 4}\right.$, Corollary 2.5] there exists a $C^{*}$-algebra isomorphism

$$
C^{*}\left(L_{2 n-1} \times{ }_{c} \mathbb{Z}_{p}\right) \cong C^{*}\left(L_{2 n-1}\right) \times_{\Lambda} \mathbb{Z}_{p} .
$$

Let $U$ be a unitary in $C^{*}\left(L_{2 n-1}\right) \times_{\Lambda} \mathbb{Z}_{p}$ such that $U^{p}=I$ and $U x U^{*}=\Lambda(x)$ for all $x \in C^{*}\left(L_{2 n-1}\right)$. For $m=0,1, \ldots, p-1$ let $Q_{m}=\frac{1}{p} \sum_{i=0}^{p-1} \theta^{i m} U^{i}$ be the spectral projection of $U$. The isomorphism (14) is given explicitly by

$$
\begin{aligned}
P_{\left(v_{i}, m\right)} & \mapsto P_{v_{i}} Q_{m}, \\
S_{\left(e_{i, j}, m\right)} & \mapsto S_{e_{i, j}} Q_{m},
\end{aligned}
$$

for $i=1, \ldots, n, j=i, \ldots, n$ and $m=0, \ldots, p-1$.

We have

$$
Q_{0}\left(C^{*}\left(L_{2 n-1}\right) \times_{\Lambda} \mathbb{Z}_{p}\right) Q_{0}=C^{*}\left(L_{2 n-1}\right)^{\Lambda} Q_{0},
$$

and the map $C^{*}\left(L_{2 n-1}\right)^{\Lambda} \rightarrow C^{*}\left(L_{2 n-1}\right)^{\Lambda} Q_{0}, x \mapsto x Q_{0}$, is a $C^{*}$-algebra isomorphism. On the other hand, the isomorphism (14) (cf. Formulae (15) and (16)) identifies $Q_{0}=\sum_{i=1}^{n} P_{v_{i}} Q_{0}$ with the projection $\sum_{i=1}^{n} P_{\left(v_{i}, 0\right)}$ in $C^{*}\left(L_{2 n-1} \times{ }_{c} \mathbb{Z}_{p}\right)$. Consequently, there is a $C^{*}$-algebra isomorphism

$$
C\left(L_{q}\left(p ; m_{1}, \ldots, m_{n}\right)\right) \cong\left(\sum_{i=1}^{n} P_{\left(v_{i}, 0\right)}\right) C^{*}\left(L_{2 n-1} \times_{c} \mathbb{Z}_{p}\right)\left(\sum_{i=1}^{n} P_{\left(v_{i}, 0\right)}\right)
$$

In the following lemma we only require that $m_{1}$ be relatively prime to $p$ and no assumptions on the remaining parameters $m_{2}, \ldots, m_{n}$ are made whatever. The lemma says that if $m_{1}$ is relatively prime to $p$ then the action $\Lambda$ is saturated, as expected.

Lemma 2.1. If $m_{1}$ is relatively prime to $p$ then for each vertex $\left(v_{k}, m\right)$ there exists a path in $L_{2 n-1} \times_{c} \mathbb{Z}_{p}$ from $\left(v_{1}, 0\right)$ to $\left(v_{k}, m\right)$. Thus, Formula 
(18) gives an isomorphism between the $C^{*}$-algebra $C\left(L_{q}\left(p ; m_{1}, \ldots, m_{n}\right)\right)$ and a full corner of $C^{*}\left(L_{2 n-1} \times_{c} \mathbb{Z}_{p}\right)$.

Proof. Let $k \in\{1, \ldots, n\}, m \in \mathbb{Z}_{p}$, and let $r$ be a positive integer such that $r m_{1}=m$ in $\mathbb{Z}_{p}$. Then

$$
\left(\left(e_{1,1}, m_{1}\right),\left(e_{1,1}, 2 m_{1}\right), \ldots,\left(e_{1,1},(r-2) m_{1}\right),\left(e_{1,1},(r-1) m_{1}\right),\left(e_{1, k}, r m_{1}\right)\right)
$$

is the desired path. Consequently,

$$
S_{\left(e_{1,1}, m_{1}\right)} S_{\left(e_{1,1}, 2 m_{1}\right)} \ldots S_{\left(e_{1,1},(r-2) m_{1}\right)} S_{\left(e_{1,1},(r-1) m_{1}\right)} S_{\left(e_{1, k}, r m_{1}\right)}
$$

is a partial isometry in $C^{*}\left(L_{2 n-1} \times_{c} \mathbb{Z}_{p}\right)$ whose domain projection equals $P_{\left(v_{k}, m\right)}$ and whose range projection is majorized by $P_{\left(v_{1}, 0\right)}$. Thus all projections $P_{\left(v_{k}, m\right)}, k=1, \ldots, n, m \in \mathbb{Z}_{p}$, belong to the ideal of $C^{*}\left(L_{2 n-1} \times{ }_{c} \mathbb{Z}_{p}\right)$ generated by $\sum_{i=1}^{n} P_{\left(v_{i}, 0\right)}$. Since $I=\sum_{k=1}^{n} \sum_{m \in \mathbb{Z}_{p}} P_{\left(v_{k}, m\right)}$ in $C^{*}\left(L_{2 n-1} \times_{c}\right.$ $\left.\mathbb{Z}_{p}\right)$, Formula (18) implies that the $C^{*}$-algebra $C\left(L_{q}\left(p ; m_{1}, \ldots, m_{n}\right)\right)$ is isomorphic to a full corner of $C^{*}\left(L_{2 n-1} \times{ }_{c} \mathbb{Z}_{p}\right)$, as claimed.

Lemma 2.1 implies that $C\left(L_{q}\left(p ; m_{1}, \ldots, m_{n}\right)\right)$ and $C^{*}\left(L_{2 n-1} \times_{c} \mathbb{Z}_{p}\right)$ are strongly Morita equivalent [23, Chapter 3]. Consequently, the $K$-groups of these two $C^{*}$-algebras are isomorphic $[\mathbf{4}, \mathbf{8}]$. In order to calculate the $K$ groups of $C^{*}\left(L_{2 n-1} \times{ }_{c} \mathbb{Z}_{p}\right)$ we assume that each of $m_{1}, \ldots, m_{n}$ is relatively prime to $p$. For short, we write $\Phi$ for the map $K_{L_{2 n-1} \times{ }_{c} \mathbb{Z}_{p}}$ which determines these $K$-groups (cf. Section 1.2). Thus, the $K_{0}$ and $K_{1}$ groups of $C^{*}\left(L_{2 n-1} \times_{c} \mathbb{Z}_{p}\right)$ are isomorphic to the cokernel and kernel, respectively, of the endomorphism $\Phi$ of the free abelian group with a basis $\left(L_{2 n-1} \times_{c} \mathbb{Z}_{p}\right)^{0}$, given by

$$
\Phi:\left(v_{i}, m\right) \mapsto\left(\sum_{j=i}^{n}\left(v_{j}, m+m_{i}\right)\right)-\left(v_{i}, m\right) .
$$

Proposition 2.2. If each of $m_{1}, \ldots, m_{n}$ is relatively prime to $p$ then

$$
K_{1}\left(C\left(L_{q}\left(p ; m_{1}, \ldots, m_{n}\right)\right)\right) \cong \mathbb{Z} .
$$

Proof. By Lemma 2.1 it sufficies to calculate the $K_{1}$-group of $C^{*}\left(L_{2 n-1} \times_{c}\right.$ $\mathbb{Z}_{p}$ ), which is isomorphic to the kernel of the map $\Phi$ from (19). Let $\lambda_{i}^{m} \in \mathbb{Z}$, for $i=1, \ldots, n$ and $m \in \mathbb{Z}_{p}$, be such that $\Phi\left(\sum_{i=1}^{n} \sum_{m \in \mathbb{Z}_{p}} \lambda_{i}^{m}\left(v_{i}, m\right)\right)=0$. This can only happen if $\sum_{j=1}^{i} \lambda_{j}^{m-m_{j}}=\lambda_{i}^{m}$ for each $i \in\{1, \ldots, n\}$ and $m \in$ $\mathbb{Z}_{p}$. Setting $i=1$, we get $\lambda_{1}^{m}=\lambda_{1}^{0}$ for all $m \in \mathbb{Z}_{p}$, because $m_{1}$ is relatively prime to $p$. Then, considering $i=2$, we get $\lambda_{1}^{0}+\lambda_{2}^{m-m_{2}}=\lambda_{2}^{m}$ for all $m \in \mathbb{Z}_{p}$. Summing this identity over $m$ we see that $\lambda_{1}^{0}=0$. Consequently, $\lambda_{2}^{m}=\lambda_{2}^{0}$ for all $m \in \mathbb{Z}_{p}$. Again, we use here the fact that $m_{2}$ is relatively prime to $p$. Continuing inductively in this manner we get $\lambda_{i}^{m}=0$ for $i=1, \ldots, n-1$ and $\lambda_{n}^{m}=\lambda_{n}^{0}$ for $m \in \mathbb{Z}_{p}$. Thus, the kernel of $\Phi$ is isomorphic to $\mathbb{Z}$, as claimed. 
It is also possible to calculate the cokernel of the map $\Phi$ and, therefore, the $K_{0}$ group of $C\left(L_{q}\left(p ; m_{1}, \ldots, m_{n}\right)\right)$. This is a simple matter if $n=2$, and we get

$$
K_{0}\left(C\left(L_{q}\left(p ; m_{1}, m_{2}\right)\right)\right) \cong \mathbb{Z} \oplus \mathbb{Z}_{p},
$$

similarly to the result of Matsumoto and Tomiyama [18]. However, if $n \geq 3$ then the calculation becomes a bit more elaborate. We illustrate with a particular case.

Proposition 2.3. If $n=3, m_{2}=m_{3}$ and both $m_{1}$ and $m_{2}$ are relatively prime to $p$ then

$$
K_{0}\left(C\left(L_{q}\left(p ; m_{1}, m_{2}, m_{3}\right)\right)\right) \cong\left\{\begin{array}{cl}
\mathbb{Z} \oplus \mathbb{Z}_{2 p} \oplus \mathbb{Z}_{\frac{p}{2}} & \text { if } p \text { is even }, \\
\mathbb{Z} \oplus \mathbb{Z}_{p} \oplus \mathbb{Z}_{p} & \text { if } p \text { is odd } .
\end{array}\right.
$$

Proof. We must determine the cokernel of $\Phi$. It is easy to see that $\left\{\left(v_{i}, 0\right) \mid\right.$ $i=1,2,3\}$ together with the range of $\Phi$ generate the entire group $\mathbb{Z}\left(L_{5} \times_{c}\right.$ $\left.\mathbb{Z}_{p}\right)^{0}$. Now let $d_{i}, \lambda_{i}^{m} \in \mathbb{Z}$, for $i=1,2,3$ and $m \in \mathbb{Z}_{p}$, be such that $\Phi\left(\sum_{i=1}^{3} \sum_{m \in \mathbb{Z}_{p}} \lambda_{i}^{m}\left(v_{i}, m\right)\right)=\sum_{i=1}^{3} d_{i}\left(v_{i}, 0\right)$. This is equivalent to

$$
\begin{aligned}
d_{i} & =\left(\sum_{j=1}^{i} \lambda_{j}^{-m_{j}}\right)-\lambda_{i}^{0}, \\
0 & =\left(\sum_{j=1}^{i} \lambda_{j}^{m-m_{j}}\right)-\lambda_{i}^{m}, \text { for } m \neq 0 .
\end{aligned}
$$

If $i=1$ then (21) gives $\lambda_{1}^{m}=\lambda_{1}^{0}$ for all $m \in \mathbb{Z}_{p}$ and then $d_{1}=0$ by (20). If $i=2$ then substituting $m=k m_{2}$ in (21), with $k=1, \ldots, p-1$, we get $\lambda_{2}^{k m_{2}}=k \lambda_{1}^{0}+\lambda_{2}^{0}$ for all $k=0, \ldots, p-1$. Then (20) yields $d_{2}=p \lambda_{1}^{0}$. If $i=3$ then substituting $m=k m_{2}$ in $(21)$, with $k=1, \ldots, p-1$, we get $\lambda_{3}^{k m_{2}}=\frac{k(k+1)}{2} \lambda_{1}^{0}+k \lambda_{2}^{0}+\lambda_{3}^{0}$ for all $k=0, \ldots, p-1$. Then (20) yields $d_{3}=\frac{p(p+1)}{2} \lambda_{1}^{0}+p \lambda_{2}^{0}$. Thus, $\left(v_{1}, 0\right)$ has infinite order in the cokernel. If $p$ is even then $\left(v_{2}, 0\right)$ and $2\left(v_{2}, 0\right)+\left(v_{3}, 0\right)$ generate a subgroup of the cokernel isomorphic to $\mathbb{Z}_{2 p} \oplus \mathbb{Z}_{\frac{p}{2}}$. If $p$ is odd then $\left(v_{2}, 0\right)$ and $\left(v_{3}, 0\right)$ generate a subgroup of the cokernel isomorphic to $\mathbb{Z}_{p} \oplus \mathbb{Z}_{p}$.

We now show that $C\left(L_{q}\left(p ; m_{1}, \ldots, m_{n}\right)\right)$ itself is isomorphic to a graph algebra. The following construction of the graph $L_{2 n-1}^{\left(p ; m_{1}, \ldots, m_{n}\right)}$ and the argument of Theorem 2.5, below, are similar to [25, Section 4 and Lemma 6]. Again, we assume that each of $m_{1}, \ldots, m_{n}$ is relatively prime to $p$.

At first we define the graph $L_{2 n-1}^{\left(p ; m_{1}, \ldots, m_{n}\right)}$, as follows: The vertices of the graph $L_{2 n-1}^{\left(p ; m_{1}, \ldots, m_{n}\right)}$ are $\left\{v_{1}, v_{2}, \ldots, v_{n}\right\}$. The edges of $L_{2 n-1}^{\left(p ; m_{1}, \ldots, m_{n}\right)}$ consist of all finite (vertex simple) paths $\alpha=\left(\left(e_{i_{1}, j_{1}}, k_{1}\right), \ldots,\left(e_{i_{r}, j_{r}}, k_{r}\right)\right)$ in $L_{2 n-1} \times_{c} \mathbb{Z}_{p}$ such that $k_{1}=m_{i_{1}}, k_{a} \neq 0$ for $a \neq r, k_{r}=0$ and $\left(v_{j_{a}}, k_{a}\right) \neq\left(v_{j_{b}}, k_{b}\right)$ for 
$a \neq b$. The source and range functions are defined as $s(\alpha)=v_{i_{1}}$ and $r(\alpha)=v_{j_{r}}$. We note that this is a finite graph without sinks, through each vertex there passes precisely one vertex simple loop (composed of a single edge), and for each pair of vertices $v_{i}, v_{j}$ there exists a path from $v_{i}$ to $v_{j}$ if and only if $i \leq j$. For example, if $n=2, p=3, m_{1}=1$ and $m_{2}=2$ then $L_{3}^{(3 ; 1,2)}$ looks as follows:

$$
L_{3}^{(3 ; 1,2)}
$$

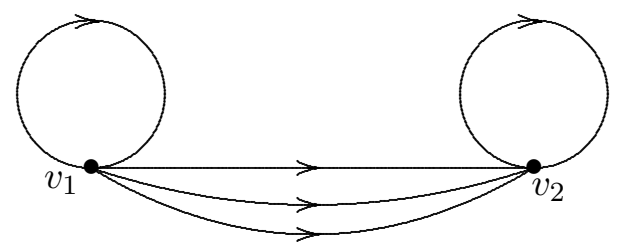

The following Lemma 2.4 essentially follows from [25, Lemma 5]. However, for the sake of completeness and reader's convenience, we give a selfcontained proof.

Lemma 2.4. If each of $m_{1}, \ldots, m_{n}$ is relatively prime to $p$ then for any $l \in\{1, \ldots, n\}$ and any $m \in \mathbb{Z}_{p}$ we have

$$
P_{\left(v_{l}, m\right)}=\sum_{\alpha} S_{\left(e_{i_{1}, j_{1}}, k_{1}\right)} \ldots S_{\left(e_{\left.i_{r}, j_{r}, k_{r}\right)}\right.} S_{\left(e_{i_{r}, j_{r}}, k_{r}\right)}^{*} \ldots S_{\left(e_{i_{1}, j_{1}}, k_{1}\right)}^{*}
$$

(in $\left.C^{*}\left(L_{2 n-1} \times{ }_{c} \mathbb{Z}_{p}\right)\right)$, where the summation extends over all $\alpha=\left(\left(e_{i_{1}, j_{1}}, k_{1}\right)\right.$, $\left.\ldots,\left(e_{i_{r}, j_{r}}, k_{r}\right)\right)$, vertex simple paths in $L_{2 n-1} \times{ }_{c} \mathbb{Z}_{p}$ such that $i_{1}=l, k_{1}-m_{i_{1}}=$ $m, k_{a} \neq 0$ for $a \neq r, k_{r}=0$ and $\left(v_{j_{a}}, k_{a}\right) \neq\left(v_{j_{b}}, k_{b}\right)$ for $a \neq b$.

Proof. For $\nu=1,2, \ldots$ we define $A_{\nu}$ to be the collection of all vertex simple paths $\alpha=\left(\left(e_{i_{1}, j_{1}}, k_{1}\right), \ldots,\left(e_{i_{r}, j_{r}}, k_{r}\right)\right)$ in $L_{2 n-1} \times_{c} \mathbb{Z}_{p}$ such that the length of $\alpha$ is not greater than $\nu$ (and nonzero), $i_{1}=l, k_{1}-m_{i_{1}}=m, k_{a} \neq 0$ for $a \neq r, k_{r}=0$ and $\left(v_{j_{a}}, k_{a}\right) \neq\left(v_{j_{b}}, k_{b}\right)$ for $a \neq b$, and let $B_{\nu}$ be the collection of all paths $\beta=\left(\left(e_{i_{1}, j_{1}}, k_{1}\right), \ldots,\left(e_{i_{r}, j_{r}}, k_{r}\right)\right)$ such that the length of $\beta$ equals $\nu, i_{1}=l, k_{1}-m_{i_{1}}=m, k_{a} \neq 0$ for $a=1, \ldots, r$ and $\left(v_{j_{a}}, k_{a}\right) \neq\left(v_{j_{b}}, k_{b}\right)$ for $a \neq b$. We show, by induction on $\nu$, that

$$
P_{\left(v_{l}, m\right)}=\sum_{\alpha \in A_{\nu}} S_{\alpha} S_{\alpha}^{*}+\sum_{\beta \in B_{\nu}} S_{\beta} S_{\beta}^{*}
$$

Indeed, the collection of all edges in $L_{2 n-1} \times_{c} \mathbb{Z}_{p}$ with source equal to $\left(v_{l}, m\right)$ is the union of $A_{1}$ and $B_{1}$. Thus, (22) holds with $\nu=1$ by virtue of (G5). Now suppose (22) holds for some $\nu$. If $\beta=\left(\left(e_{i_{1}, j_{1}}, k_{1}\right), \ldots,\left(e_{i_{r}, j_{r}}, k_{r}\right)\right)$ in $B_{\nu}$, then applying Condition (G5) at the range vertex of $\beta$, equal to $\left(v_{j_{r}}, k_{r}\right)$, we get

$$
S_{\beta} S_{\beta}^{*}=S_{\beta} P_{\left(v_{j_{r}}, k_{r}\right)} S_{\beta}^{*}=\sum_{d=j_{r}}^{n} S_{\beta} S_{\left(e_{j_{r}, d}, k_{r}+m_{j_{r}}\right)} S_{\left(e_{j_{r}, d}, k_{r}+m_{j_{r}}\right)}^{*} S_{\beta}^{*}
$$


Let $\beta^{\prime}=\left(\left(e_{i_{1}, j_{1}}, k_{1}\right), \ldots,\left(e_{i_{r}, j_{r}}, k_{r}\right),\left(e_{j_{r}, d}, k_{r}+m_{j_{r}}\right)\right)$. We claim that $\left(v_{d}, k_{r}+\right.$ $\left.m_{j_{r}}\right) \neq\left(v_{j_{a}}, k_{a}\right)$ for $a=1, \ldots, r$. This is obvious if $d \neq j_{r}$. For $d=j_{r}$ let $b$ be the smallest index such that $j_{b}=j_{r}$. Since $\beta$ is a path we have $j_{b}=j_{b+1}=\cdots=j_{r}$ and $k_{b+h}=k_{b}+h m_{j_{b}}$ for $h=1, \ldots, r-b$. Since $m_{j_{b}}$ is relatively prime to $p$ it follows that $k_{r}+m_{j_{r}} \notin\left\{k_{b}, \ldots, k_{r}\right\}$, as claimed. Thus $\beta^{\prime} \in\left(A_{\nu+1} \backslash A_{\nu}\right) \cup B_{\nu+1}$. Consequently, from the inductive hypothesis, Formula (23) and the above discussion we get

$$
\begin{aligned}
P_{\left(v_{l}, m\right)} & =\sum_{\alpha \in A_{\nu}} S_{\alpha} S_{\alpha}^{*}+\sum_{\beta \in B_{\nu}} S_{\beta} S_{\beta}^{*} \\
& =\sum_{\alpha \in A_{\nu}} S_{\alpha} S_{\alpha}^{*}+\sum_{\beta^{\prime} \in\left(A_{\nu+1} \backslash A_{\nu}\right)} S_{\beta^{\prime}} S_{\beta^{\prime}}^{*}+\sum_{\beta^{\prime} \in B_{\nu+1}} S_{\beta^{\prime}} S_{\beta^{\prime}}^{*} \\
& =\sum_{\alpha \in A_{\nu+1}} S_{\alpha} S_{\alpha}^{*}+\sum_{\beta \in B_{\nu+1}} S_{\beta} S_{\beta}^{*},
\end{aligned}
$$

and the inductive step follows.

Since $L_{2 n-1} \times{ }_{c} \mathbb{Z}_{p}$ is a finite graph there exists a $\nu$ large enough so that $B_{\nu}=\emptyset$. With this $\nu$ Formula (22) gives the lemma.

Theorem 2.5. If each of the numbers $m_{1}, \ldots, m_{n}$ is relatively prime to $p$ then the $C^{*}$-algebra $C\left(L_{q}\left(p ; m_{1}, \ldots, m_{n}\right)\right)$ is isomorphic to $C^{*}\left(L_{2 n-1}^{\left(p ; m_{1}, \ldots, m_{n}\right)}\right)$.

Proof. At first we observe that there exists a $C^{*}$-algebra homomorphism

$$
\psi: C^{*}\left(L_{2 n-1}^{\left(p ; m_{1}, \ldots, m_{n}\right)}\right) \rightarrow\left(\sum_{i=1}^{n} P_{\left(v_{i}, 0\right)}\right) C^{*}\left(L_{2 n-1} \times_{c} \mathbb{Z}_{p}\right)\left(\sum_{i=1}^{n} P_{\left(v_{i}, 0\right)}\right)
$$

such that

$$
\begin{aligned}
\psi: P_{v_{l}} & \mapsto P_{\left(v_{l}, 0\right)}, \\
\psi: S_{\alpha} & \mapsto S_{\left(e_{i_{1}, j_{1}}, k_{1}\right)} S_{\left(e_{i_{2}, j_{2}}, k_{2}\right)} \ldots S_{\left(e_{i_{r}, j_{r}}, k_{r}\right)},
\end{aligned}
$$

for all $l=1, \ldots, n$ and for all $\alpha=\left(\left(e_{i_{1}, j_{1}}, k_{1}\right), \ldots,\left(e_{i_{r}, j_{r}}, k_{r}\right)\right)$, vertex simple paths in $L_{2 n-1} \times_{c} \mathbb{Z}_{p}$ such that $k_{1}=m_{i_{1}}, k_{a} \neq 0$ for $a \neq r, k_{r}=$ 0 and $\left(v_{j_{a}}, k_{a}\right) \neq\left(v_{j_{b}}, k_{b}\right)$ for $a \neq b$. Due to the universal property of $C^{*}\left(L_{2 n-1}^{\left(p ; m_{1}, \ldots, m_{n}\right)}\right)$, to this end it sufficies to verify that the elements $\left\{\psi\left(P_{v_{l}}\right)\right.$, $\left.\psi\left(S_{\alpha}\right)\right\}$ of $C^{*}\left(L_{2 n-1} \times_{c} \mathbb{Z}_{p}\right)$ satisfy Conditions (G1)-(G5) for the graph $L_{2 n-1}^{\left(p ; m_{1}, \ldots, m_{n}\right)}$. But it is obvious that Conditions (G1)-(G4) are satisfied, and Condition (G5) is equivalent to Lemma 2.4 with $m=0$.

For surjectivity of $\psi$ it sufficies to show that:

(i) If $\alpha$ is a path in $L_{2 n-1} \times_{c} \mathbb{Z}_{p}$ such that both $s(\alpha)$ and $r(\alpha)$ are in $\left\{\left(v_{i}, 0\right) \mid i=1, \ldots, n\right\}$ then $S_{\alpha}$ belongs to the range of $\psi$.

(ii) If $\alpha, \beta$ are two paths such that $r(\alpha)=r(\beta)$ and both $s(\alpha)$ and $s(\beta)$ are in $\left\{\left(v_{i}, 0\right) \mid i=1, \ldots, n\right\}$ then $S_{\alpha} S_{\beta}^{*}$ belongs to the range of $\psi$. 
To this end we first note that any loop in $L_{2 n-1} \times_{c} \mathbb{Z}_{p}$ must pass through a vertex of the form $\left(v_{i}, 0\right)$. Now let $\alpha=\left(\left(e_{i_{1}, j_{1}}, k_{1}\right), \ldots,\left(e_{i_{r}, j_{r}}, k_{r}\right)\right)$ be a path as in (i). Let $a_{1}<a_{2}<\cdots<a_{\nu}=r$ be all the indices for which $k_{a_{t}}=0$. We also set $a_{0}=0$. For each $t=1, \ldots, \nu$ the path $\alpha_{t}=\left(\left(e_{i_{1+a_{t-1}}, j_{1+a_{t-1}}}, k_{1+a_{t-1}}\right), \ldots,\left(e_{i_{a_{t}}, j_{a_{t}}}, k_{a_{t}}\right)\right)$ is an edge of the graph $L_{2 n-1}^{\left(p ; m_{1}, \ldots, m_{n}\right)}$. Hence, $S_{\alpha}=S_{\alpha_{1}} \ldots S_{\alpha_{\nu}}$ belongs to the range of $\psi$, since each $S_{\alpha_{t}}$ does. Now let $\alpha$ and $\beta$ be two paths as in (ii). Let $\alpha=\left(\left(e_{i_{1}, j_{1}}, k_{1}\right), \ldots\right.$, $\left.\left(e_{i_{r}, j_{r}}, k_{r}\right)\right)$. By virtue of Part (i) it sufficies to consider the case $k_{r} \neq$ 0 . Let $\mu$ be the greatest index such that $k_{\mu}=0$, or 0 if such an index does not exist. We set $\alpha_{1}=\left(\left(e_{i_{1}, j_{1}}, k_{1}\right), \ldots,\left(e_{i_{\mu}, j_{\mu}}, k_{\mu}\right)\right)$ and $\alpha_{2}=$ $\left(\left(e_{i_{\mu+1}, j_{\mu+1}}, k_{\mu+1}\right), \ldots,\left(e_{i_{r}, j_{r}}, k_{r}\right)\right)$. We have $S_{\alpha}=S_{\alpha_{1}} S_{\alpha_{2}}$ and $S_{\alpha_{1}}$ is in the range of $\psi$ by Part (i) (if $\mu=0$ then $\alpha_{1}=\emptyset$ and $S_{\alpha_{1}}=I$ ). Furthermore, for $\mu+1 \leq a, b \leq r$ we have $\left(v_{j_{a}}, k_{a}\right) \neq\left(v_{j_{b}}, k_{b}\right)$ if $a \neq b$. We have an analogous factorization $S_{\beta}=S_{\beta_{1}} S_{\beta_{2}}$, with $S_{\beta_{1}}$ in the range of $\psi$. Let $P_{\left(v_{r}, k_{r}\right)}=\sum_{x} S_{x} S_{x}^{*}$ be the decomposition as in Lemma 2.4. Then we have

$$
S_{\alpha} S_{\beta}=S_{\alpha_{1}} S_{\alpha_{2}} P_{\left(v_{r}, k_{r}\right)} S_{\beta_{2}}^{*} S_{\beta_{1}}^{*}=\sum_{x} S_{\alpha_{1}} S_{\alpha_{2}} S_{x} S_{x}^{*} S_{\beta_{2}}^{*} S_{\beta_{1}}^{*}
$$

Consequently, $S_{\alpha} S_{\beta}$ belongs to the range of $\psi$, since $S_{\alpha_{1}}, S_{\beta_{1}}$ and all $S_{\alpha_{2}} S_{x}$ and $S_{\beta_{2}} S_{x}$ do. This completes the proof of surjectivity of $\psi$.

Now we show that the homomorphism $\psi$ is injective. Our argument is essentially the same as in [5, Remark 3]. Since for each $i \in\{1, \ldots, n-1\}$ there exists a path from $v_{i}$ to $v_{n}$, the ideal $J$ of $C^{*}\left(L_{2 n-1}^{\left(p ; m_{1}, \ldots, m_{n}\right)}\right)$ generated by $P_{v_{n}}$ is essential by Lemma 1.1. Thus, it sufficies to show that $J \cap \operatorname{ker}(\psi)=$ $\{0\}$. To this end, we notice that in the graph $L_{2 n-1}^{\left(p ; m_{1}, \ldots, m_{n}\right)}$ the vertex $v_{n}$ emits a unique edge, which we call $e$, and the range of this edge is $v_{n}$. Since there are infinitely many paths from other vertices to $v_{n}$ it follows (cf. [15] and [5, Remark 3]) that

$$
J \cong P_{v_{n}} J P_{v_{n}} \otimes \mathcal{K}=C^{*}\left(S_{e}\right) \otimes \mathcal{K} \cong C(\mathbb{T}) \otimes \mathcal{K} .
$$

Hence, in order to prove injectivity of $\psi$ it sufficies to show that $C^{*}\left(S_{e}\right) \cap$ $\operatorname{ker}(\psi)=\{0\}$. This follows from the fact that

$$
\psi\left(S_{e}\right)=S_{\left(e_{n, n}, m_{n}\right)} S_{\left(e_{n, n}, 2 m_{n}\right)} \ldots S_{\left(e_{n, n}, p m_{n}\right)}
$$

is a partial unitary with full spectrum (cf. [15]).

With help of Theorem 2.5 it is easy to determine the ideal structure of $C\left(L_{q}\left(p ; m_{1}, \ldots, m_{n}\right)\right)$. For example, we have seen in the proof of Theorem 2.5 that the ideal of $C^{*}\left(L_{2 n-1}^{\left(p ; m_{1}, \ldots, m_{n}\right)}\right)$ generated by $P_{v_{n}}$ is isomorphic to $C(\mathbb{T}) \otimes \mathcal{K}$. The corresponding quotient is $C^{*}\left(L_{2 n-3}^{\left(p ; m_{1}, \ldots, m_{n-1}\right)}\right)$, and this $C^{*}$-algebra is in turn isomorphic to $C\left(L_{q}\left(p ; m_{1}, \ldots, m_{n-1}\right)\right)$. Thus, there 
exists an exact sequence

(24)

$$
0 \rightarrow C(\mathbb{T}) \otimes \mathcal{K} \rightarrow C\left(L_{q}\left(p ; m_{1}, \ldots, m_{n}\right)\right) \rightarrow C\left(L_{q}\left(p ; m_{1}, \ldots, m_{n-1}\right)\right) \rightarrow 0 .
$$

Using the exact sequence (24) or the general results about graph algebras, outlined in Section 1.3, it is easy to understand the primitive spectrum of $C\left(L_{q}\left(p ; m_{1}, \ldots, m_{n}\right)\right)$. Therefore, we omit the proof of the following proposition:

Proposition 2.6. If each of $m_{1}, \ldots, m_{n}$ is relatively prime to $p$ then the primitive ideal space of $C\left(L_{q}\left(p ; m_{1}, \ldots, m_{n}\right)\right)$ consists of $n$ disjoint copies $C_{1}, \ldots, C_{n}$ of the circle. The hull-kernel topology restricted to each of the circles coincides with the natural one. The closure of a point in $C_{k}$ contains $C_{1} \cup \cdots \cup C_{k-1}$. Thus, $\operatorname{Prim}\left(C\left(L_{q}\left(p ; m_{1}, \ldots, m_{n}\right)\right)\right)$ and $\operatorname{Prim}\left(C\left(S_{q}^{2 n-1}\right)\right)$ are homeomorphic (cf. [11, Section 4.1]).

Concluding remarks. For a fixed integer $p \geq 2$ the infinite lens space $L(p ; \infty)$ is defined as the inductive limit of the lens spaces $L\left(p ; 1_{n}\right)$, corresponding to the natural imbeddings $L\left(p ; 1_{n}\right) \hookrightarrow L\left(p ; 1_{n+1}\right)$. (If $m_{1}=\cdots=$ $m_{n}=1$ then we simply write $L\left(p ; 1_{n}\right)$ instead of $L(p ; 1, \ldots, 1)$.) It turns out that $L(p ; \infty)$ is identical with the Eilenberg-MacLane space of type $\left(\mathbb{Z}_{p}, 1\right)$ $[3]$.

The results of the previous section lead to quantum versions of this classical topological setting. Namely, the inclusion $L\left(p ; 1_{n}\right) \hookrightarrow L\left(p ; 1_{n+1}\right)$ corresponds to the surjective homomorphism $\widetilde{\theta}_{n+1}: C\left(L_{q}\left(p ; 1_{n+1}\right)\right) \rightarrow C\left(L_{q}\left(p ; 1_{n}\right)\right)$ such that the kernel of $\widetilde{\theta}_{n+1}$ is generated by $z_{n+1} z_{n+1}^{*}$. Consequently, the quantum infinite lens space, or the quantum Eilenberg-MacLane space of type $\left(\mathbb{Z}_{p}, 1\right)$, may be defined as the inverse limit

$$
C\left(L_{q}(p ; \infty)\right)=\lim _{\longleftarrow}\left(C\left(L_{q}\left(p ; 1_{n}\right)\right), \widetilde{\theta}_{n}\right) .
$$

Under the isomorphisms $C\left(L_{q}\left(p ; 1_{k}\right)\right) \cong C^{*}\left(L_{2 k-1}^{(p)}\right)$ (if $m_{1}=\cdots=m_{n}=$ 1 then we simply write $L_{2 n-1}^{(p)}$ instead of $L_{2 n-1}^{(p ; 1, \ldots, 1)}$ ), described in Theorem 2.5, the homomorphism $\widetilde{\theta}_{n+1}$ is carried onto a surjective $C^{*}$-algebra homomorphism $\theta_{n+1}: C^{*}\left(L_{2 n+1}^{(p)}\right) \rightarrow C^{*}\left(L_{2 n-1}^{(p)}\right)$, whose kernel is generated by the projection $P_{v_{n+1}}$. Thus, we have the $C^{*}$-algebra isomorphism

$$
C\left(L_{q}(p ; \infty)\right) \cong \lim _{\longleftarrow}\left(C^{*}\left(L_{2 n-1}^{(p)}\right), \theta_{n}\right) .
$$

It is not difficult to see, and we omit the details, that this inverse limit itself may be realized as the graph algebra $C^{*}\left(L_{\infty}^{(p)}\right)$. The graph $L_{\infty}^{(p)}$ is the increasing limit of the graphs $L_{2 n-1}^{(p)}$, corresponding to the natural imbeddings $L_{2 n-1}^{(p)} \hookrightarrow L_{2 n+1}^{(p)}$ such that the $v_{i}$ vertex in $L_{2 n-1}^{(p)}$ is identified with 
the $v_{i}$ vertex in $L_{2 n+1}^{(p)}$, and the edges from $v_{i}$ to $v_{j}$ in $L_{2 n-1}^{(p)}$ are bijectively identified with the edges from $v_{i}$ to $v_{j}$ in $L_{2 n+1}^{(p)}$. The graph $L_{\infty}^{(p)}$ has infinitely many vertices $\left\{v_{1}, v_{2} \ldots\right\}$, and for each pair $i \leq j$ there exists at least one edge from $v_{i}$ to $v_{j}$. These two properties imply that $C^{*}\left(L_{\infty}^{(p)}\right)$ is a primitive, purely infinite $C^{*}$-algebra (but not simple) [1]. Furthermore, $K_{0}\left(C^{*}\left(L_{\infty}^{(p)}\right)\right) \cong \bigoplus^{\infty} \mathbb{Z}$ and $K_{1}\left(C^{*}\left(L_{\infty}^{(p)}\right)\right)=0[\mathbf{2 2}, \mathbf{7}]$.

\section{References}

[1] T. Bates, J.H. Hong, I. Raeburn and W. Szymański, The ideal structure of the $C^{*}$ algebras of infinite graphs, Illinois J. Math., 46 (2002), 1159-1176.

[2] T. Bates, D. Pask, I. Raeburn and W. Szymański, The $C^{*}$-algebras of row-finite graphs, New York J. Math., 6 (2000), 307-324, MR 2001k:46084, Zbl 0976.46041.

[3] R. Bott and L.W. Tu, Differential Forms in Algebraic Topology, Graduate Texts in Math., 82, Springer-Verlag, New York, 1982, MR 83i:57016, Zbl 0496.55001.

[4] L.G. Brown, Stable isomorphism of hereditary subalgebras of $C^{*}$-algebras, Pacific J. Math., 71 (1977), 335-348, MR 56 \#12894, Zbl 0362.46042.

[5] J. Cuntz, A class of $C^{*}$-algebras and topological Markov chains II: Reducible chains and the Ext-functor for $C^{*}$-algebras, Invent. Math., 63 (1981), 25-40, MR 82f:46073b, Zbl 0461.46047.

[6] J. Cuntz and W. Krieger, A class of $C^{*}$-algebras and topological Markov chains, Invent. Math., 56 (1980), 251-268, MR 82f:46073a, Zbl 0434.46045.

[7] D. Drinen and M. Tomforde, Computing K-theory and Ext for graph $C^{*}$-algebras, Illinois J. Math., 46(1) (2002), 81-91, MR 1936076.

[8] R. Exel, A Fredholm operator approach to Morita equivalence, K-Theory, 7 (1993), 285-308, MR 94h:46107, Zbl 0792.46051.

[9] N.J. Fowler, M. Laca and I. Raeburn, The $C^{*}$-algebras of infinite graphs, Proc. Amer. Math. Soc., 128 (2000), 2319-2327, MR 2000k:46079, Zbl 0956.46035.

[10] J.H. Hong, The ideal structure of graph algebras, in 'Proceedings of the USA-Japan Seminar on Operator Algebras and Applications' (Fukuoka, 1999), to appear.

[11] J.H. Hong and W. Szymański, Quantum spheres and projective spaces as graph algebras, Commun. Math. Phys., 232 (2002), 157-188, MR 1942860.

[12] A. an Huef and I. Raeburn, The ideal structure of Cuntz-Krieger algebras, Ergodic Theory Dynam. Systems, 17 (1997), 611-624, MR 98k:46098, Zbl 0886.46061.

[13] M. Karoubi, K-Theory. An Introduction, Springer-Verlag, Berlin, 1978, MR 58 \#7605, Zbl 0382.55002.

[14] A. Kumjian and D. Pask, $C^{*}$-algebras of directed graphs and group actions, Ergodic Theory Dynam. Systems, 19 (1999), 1503-1519, MR 2000m:46125, Zbl 0949.46034.

[15] A. Kumjian, D. Pask and I. Raeburn, Cuntz-Krieger algebras of directed graphs, Pacific J. Math., 184 (1998), 161-174, MR 99i:46049, Zbl 0917.46056.

[16] A. Kumjian, D. Pask, I. Raeburn and J. Renault, Graphs, groupoids, and CuntzKrieger algebras, J. Funct. Anal., 144 (1997), 505-541, MR 98g:46083. 
[17] E.Ch. Lance, The compact quantum group $S O(3)_{q}$, J. Operator Theory, 40 (1998), 295-307, MR 99h:46134, Zbl 1002.46043.

[18] K. Matsumoto and J. Tomiyama, Noncommutative lens spaces, J. Math. Soc. Japan, 44 (1992), 13-41, MR 93g:46068, Zbl 0731.46038.

[19] G. Nagy, On the Haar measure of the quantum $S U(N)$ group, Commun. Math. Phys., 153 (1993), 217-228, MR 94e:46124, Zbl 0779.17015.

[20] P. Podleś, Symmetries of quantum spaces. Subgroups and quotient spaces of quantum $S U(2)$ and $S O(3)$ groups, Commun. Math. Phys., 170 (1995), 1-20, MR 96j:58013, Zbl 0853.46074.

[21] W. Pusz and S.L. Woronowicz, Twisted second quantization, Rep. Math. Phys., 27 (1989), 231-257, MR 93c:81082, Zbl 0707.47039.

[22] I. Raeburn and W. Szymański, Cuntz-Krieger algebras of infinite graphs and matrices, Trans. Amer. Math. Soc., to appear.

[23] I. Raeburn and D.P. Williams, Morita Equivalence and Continuous-Trace $C^{*}$ Algebras, Math. Surveys and Monographs, 60, Amer. Math. Soc., Providence, 1998, MR 2000c:46108, Zbl 0922.46050.

[24] W. Szymański, On semiprojectivity of $C^{*}$-algebras of directed graphs, Proc. Amer. Math. Soc., 130 (2002), 1391-1399, MR 2003a:46083.

[25] - The range of $K$-invariants for $C^{*}$-algebras of infinite graphs, Indiana Univ. Math. J., 51 (2002), 239-249, MR 2003b:46077.

[26] L.L. Vaksman and Y.S. Soibelman, Algebra of functions on the quantum $S U(n+1)$ group and odd dimensional quantum spheres, Algebra i Analiz, 2 (1990), 101-120, MR 92e:58021, Zbl 0751.46048.

[27] S.L. Woronowicz, Twisted SU(2) group. An example of a non-commutative differential calculus, Publ. Res. Inst. Math. Sci., 23 (1987), 117-181, MR 88h:46130, Zbl 0676.46050 .

Received March 13, 2001 and revised November 4, 2002.

This work was supported by grant No. R04-2001-000-00117-0 from Korea Science \& Engineering Foundation.

\author{
Applied Mathematics \\ Korea Maritime University \\ BUSAN 606-791 \\ SOUTh KoreA \\ E-mail address: hongjh@hanara.kmaritime.ac.kr \\ MATHEMATICS \\ The University of Newcastle \\ Callaghan, NSW 2308 \\ Australia \\ E-mail address: wojciech@frey.newcastle.edu.au
}

